\title{
Real Time Optimization for Quality Control of Batch Thermal Sterilization of Prepackaged Foods
}

\author{
Antonio A. Alonso, Ana Arias-Méndez, Eva Balsa-Canto, Míriam R. \\ García, Juan I. Molina, Carlos Vilas, Marcos Villafín
}

\begin{abstract}
In this contribution, we present a distributed decision-making architecture for control to optimally command thermal sterilization, despite process uncertainty or unexpected process disturbances. The control structure combines in a synchronous way modeling and simulation environments with efficient system identification and dynamic optimization tools and methods. Process simulation provides a complete dynamic description of the current status of the operation, including the evolution of temperature and pressure in the retort unit as well as temporal and spatial distribution of temperature and quality or safety parameters within the product. Such virtual representation will be regularly confronted with plant measurements to quantify the degree of discrepancy (uncertainty) between real plant and models and react accordingly when such discrepancy becomes unacceptable by re-estimating plant parameters, either during the cycle or from batch to batch. The virtual plant will be also accessed by the regulatory system as well as the dynamic optimization module. In the first instance to estimate unmeasured states related with the product status (e.g. temperature in the product or lethality) under feed-back control. In the second, to continuously recompute optimal cycle profiles so to respond to unexpected disturbances or deviations from the prescribed safety constraints while maximizing quality attributes. Experimental evidence of the complete control system performance will be given on the operation of a pilot plant prototype.
\end{abstract}

Keywords: Thermal Sterilization, Real Time Optimization, On-line Control, Parameter Identification, Optimal Quality Control, Food Safety Control 


\section{Introduction}

The primary goal of thermal sterilization is the destruction or inactivation by the action of heat of potentially harmful spores or microorganisms that might be present in the foodstuff. To that purpose the prepackaged product is subject to a prescribed temperature-time profile calculated so to ensure the reduction of spores or microorganisms to levels that are harmless for human consumption, even if stored during large periods of time.

Thermal sterilization is a particularly demanding operation both in terms of energy consumption and process time. In addition, it may lead to significant product quality losses if not operated properly, as nutrients or sensory parameters (color or texture for instance) can be adversely affected by the thermal treatment. In fact, and apart from those situations which might demand some desired "cooking effect" that requires heating beyond that needed for food safety (Teixeira \& Tucker, 1997), thermal processes will have a detrimental influence on product quality (real or perceived).

The effect of the time-temperature profiles on the operation costs and product quality, was quite well understood in the past. In the same way, the adverse effect of disturbances in the process, such as those in steam supply that could lead to rejection of the batch or reprocessing were studied also extensively (Teixeira \& Tucker, 1997).

As early as in the 70's, research efforts in thermal sterilization were directed to compute optimal retort temperature profiles. The formulation of optimal control problems for thermal processing typically made use of retort temperature as the control variable (Teixeira et al., 1975). Saguy \& Karel (1979) and Nadkarni \& Hatton (1985) were among the first to formulate and solve an optimal control problem to maximize quality (in this case thiamine retention) in thermal processing for conduction-heated foodstuff. The resulting optimal profiles usually consisted of retort temperatures hitting upper and lower constraints (bang-bang control). The study of optimal control policies for thermal sterilization was extended by Banga et al. (1991) to a number of optimal control problems involving minimization of time and energy, in addition to maximizing product quality, subject to a constraint on the minimum final lethality. As a result, a set of constant and variable retort temperature profiles were proposed. Its implementation however required knowledge of the retort dynamics which in turn demanded advanced controllers (Alonso et al., 1997, 1998). A review on optimization methods to compute optimal temperature profiles can be found in Durance (1997). 
Based on a model predictive control paradigm, Chalabi et al. (1999) proposed the on-line implementation of an optimal control solution for thermal sterilization. However, the controller only made use of the heat transfer model for the product without consideration of the dynamics of the retort.

Similar problems were considered by Balsa-Canto et al. (2002b) to explore the potentialities in dynamic optimization of reduced order models based on POD (Proper Orthogonal Decomposition) expansions of the product heat transfer equation. More recently, optimal control problems for thermal sterilization oriented to quality maximization have been undertaken in the context of multi-objective optimization (Erdogdu \& Balaban, 2003; Sendin et al., 2010; Abakarov et al., 2009), local and global optimization algorithms (Chen \& Ramaswamy, 2002; Ansorena \& Salvadori, 2011; Miri et al., 2008; Enitan \& Adeyemo, 2011), and adaptive search techniques (Miri et al., 2008; Simpson et al., 2008; Simpson \& Abakarov, 2011).

Unfortunately, in computing optimal policies for thermal processing most works disregard the dynamics of the retort, including the regulatory layer. This in many instances may lead to unfeasible retort temperature profiles or processes undergoing high energy consumptions, as observed by Alonso et al. $(1997,1998)$ and confirmed in the present contribution. These authors developed model based and adaptive control schemes to implement optimal retort temperature profiles that minimized the adverse effects produced by temperature deviations.

Typically, what is meant by on-line retort control is the implementation of logic decision charts which in the event of given deviations in temperature or pressure will propose alternative heating profiles (usually constant timetemperature) compliant with a process requirement. This usually reduces to a minimum required lethality and does not pay attention to product quality. As discussed in Teixeira \& Tucker (1997); Akterian (1999), and more recently by Simpson et al. (2007a,b); Chen et al. (2008), system decisions are based on real-time recording of microbiological lethality. Monitoring can be done either from direct temperature measurements at the center of the can or predicted by mathematical models that describe the temperature distribution within the can (and thus lethality at the cold point) based on the actual retort temperature. Monitoring systems may be completed with a set of decision rules aimed at selecting a given constant retort temperature to track, in the event of process deviations, so to assure a minimum lethality. More advanced rules are computed by taking into account previous temperature history but usually assume constant temperature until the end of the cycle (Simpson 
et al., 2007b). Nonetheless, to the best of our knowledge, no provisions for process re-optimization have been incorporated in these implementations yet.

Nowadays and mainly driven by consumer demand, food industries are making significant efforts to ensure that their products will attain the highest possible quality without compromising safety standards (this meaning real or perceived quality). This in turn has a direct impact on the price the consumer is willing to pay for the products and therefore on the company turnover, which in addition will influence the company's strategic position in the market (Miri et al., 2008).

Good examples include the so-called gourmet preparations or products packaged in new formats as it is the case of flexible packs (Simpson et al., 2004). While over-processing will result in unacceptable quality losses, safety constraint satisfaction near its very lower limit becomes more and more critical (Martins et al., 2008). In a related way, processing flexible packages or retortable pouches demands strict pressure control during the whole cycle in order to avoid sharp pressure drops which might damage containers, thereby favoring product recontamination (Alonso et al., 1997).

All this calls for novel operation modes for thermal sterilization, optimally adapted to product safety and quality specifications even in the presence of faults (e.g. temporary down-fall of the steam supply). Such aim, however, is hampered by a number of obstacles which difficult on-line optimal decision making. Among those, one must point out the complexity of any reliable description of a process (a model) which being essentially batch, may involve a variety of phenomena associated to bio-transformations as well as mass and energy transfer mechanisms with its diversity of spatial and temporal scales (Koribilli et al., 2011). From a control point of view, advanced model-based predictive control methods (MPC) are the appropriate framework capable of producing optimal temperature and pressure profiles on an uncertain environment (Camacho \& Bordóns, 1995; Banga et al., 2008). In particular, the MPC approach has been successfully applied in the context of batch processes in the food industry, (e.g. Flores-Cerrillo \& MacGregor, 2005; Kurtanjek, 2008).

Pursuing such direction, this contribution presents a robust model based decision-making architecture to optimally command thermal processing operation, despite process uncertainty or unexpected process disturbances. Some novel features of the present control system must be underlined as they surmount the main obstacles to real time implementation. These include, the 


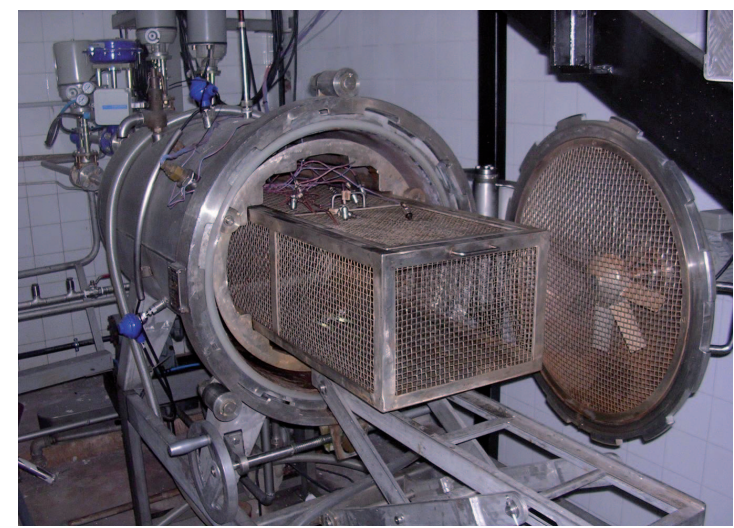

Figure 1: A detail of the pilot plant batch steam retort employed for the control experiments. It can be distinguished in the picture a pneumatic valve at the upper left corner and two of the three PT100 located around the vessel.

availability of efficient (i.e. fast) yet accurate simulations of the process, and the possible infeasibility of the resulting policies. The first issue has been overcome by taking advantage of highly efficient model reduction techniques for partial differential equations (which in our case would be associated to the heat transfer model within the product) based on spectral methods, and particularly on the POD method (see for example, Sirovich, 1987; Balsa-Canto et al., 2002a). On the other hand, infeasibility of the resulting optimal retort temperature profile has been overcome by including within the dynamic optimization problem the dynamics of the thermal unit itself. Such extensions to previous work will be described in detail in the paper. Finally, experimental evidence of the performance exhibited by the proposed control configuration will be given and discussed, constituting the main contribution of the work.

\section{Materials and methods}

\subsection{Thermal plant description}

This study considers the problem of optimally controlling the pilot plant steam batch thermal sterilization unit depicted in Figure 1. A typical sterilization cycle is usually divided in three stages known as venting, heating and cooling (Lopez, 1987). During the first stage (venting) air is swept off the retort with steam to ensure that the heating medium contains just saturated steam. To that purpose, bleeder and drain valves are kept fully open while steam is being injected in the retort until its pressure equals that 


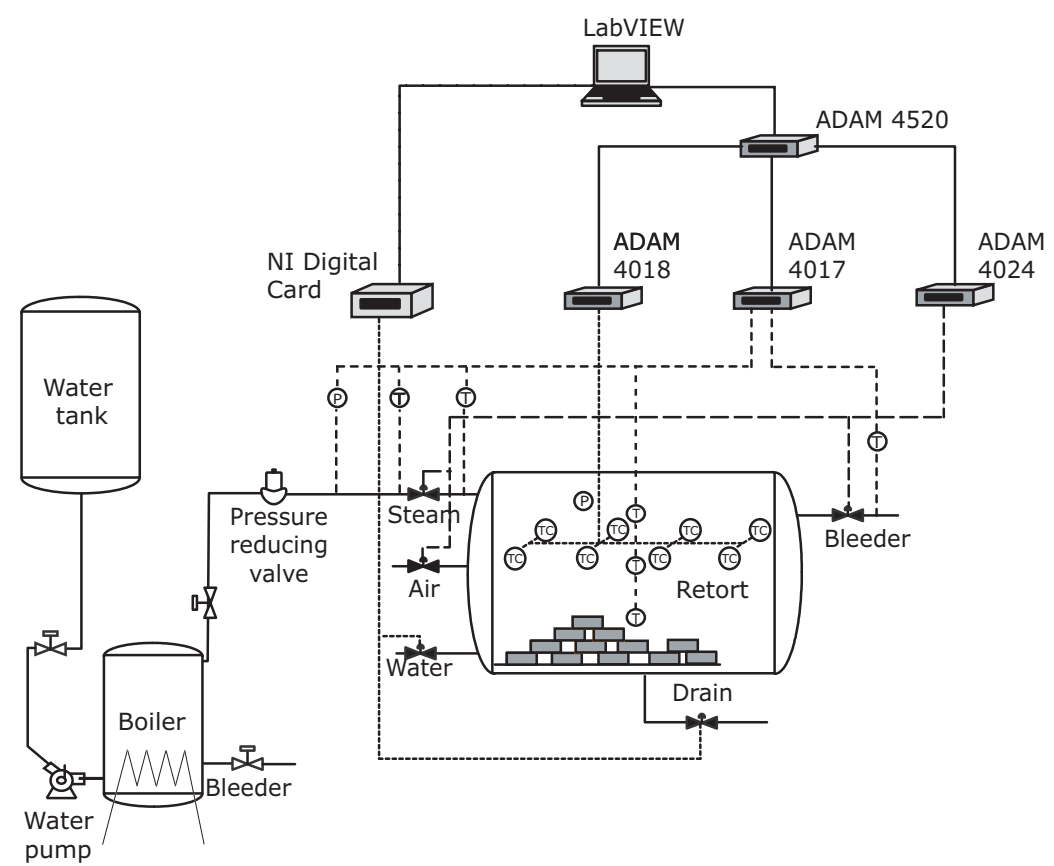

Figure 2: A diagram of the thermal processing unit and the computer acquisition and control system. The thermal system includes the steam supply line and the sterilization retort. ADAM modules (Advantech) are employed to record temperature and pressure in the vessel and send it to the computer. Valve positions are transmitted to the actuation elements by the computer via a digital card (National Instruments).

of water vapor pressure at the retort temperature. The cycle continues with the heating stage, which is where sterilization mainly takes place, by keeping the product under a given (constant or variable) retort temperature profile associated to a prescribed lethality at the coldest point within the product. Finally, and in order to reduce over-processing, the product is cooled down to room temperature by water flowing into the process vessel. Typically, the food product will be cooled with water either by immersion or a shower over the retort load. In order to compensate for any pressure drop that results from vapor condensation, overpressure is produced in the retort by air injection, right before water enters.

The sterilization unit used in this work consists of a 350 liters steel vessel with a product storage grid box with rotary capacity and a fan to ensure temperature homogeneity during the heating sterilization cycle (see Figure 1). As presented in Figure 2, the retort unit is equipped with pneumatic 
valves, used to control temperature and pressure along the sterilization cycle by regulating steam, air input and bleeder streams.

Two motorized valves, one to set up cooling water flow and the other dedicated to drain water from the vessel (either condensate or cooling water) complete the set of actuators. Pressure and temperature in the retort are measured by a number of ST18 piezo-resistive and PT100 temperature transmitters, indicated as $P$ and $T$ in Figure 2. These devices are installed both along the steam supply line (from boiler to steam valve) and in the retort. A set of thermocouples (Ecklund type T) (indicated as $T C$ in the figure) is also employed to measure temperature at different locations within the product. As represented in the figure, signals are processed by ADAM modules and recorded in the monitoring and control computer.

The monitorization and control interface has been developed in Labview ${ }^{1}$, a flexible acquisition and control software environment to record temperature, pressure and valve position on the one hand, and to implement control actions on the other. In the present study, data sampling and actuations were carried out at intervals of 5 seconds. The environment accepts the integration of modular and Matlab compatible process simulators such as EcosimPro ${ }^{2}$, with optimization software. This allows on-line prediction of the evolution of safety and quality parameters such as lethality or nutrient retention, for instance. A diagram showing the information flow between the process and the different elements that constitute the control system is presented in Figure 3. The input-output data transfer, monitoring and control, simulation and optimization units constitute the building blocks of the real time optimal controller. Its elements and interconnections will be explained in more detail in Section 2.3.

\subsection{Mathematical description of the process}

Any model based control architecture requires a reliable dynamic representation of the plant. In our case the plant comprises two interrelated models: one to describe temperature and pressure evolution in the retort unit and the other to describe the evolution of safety and quality product properties during the thermal treatment. In the case of solid foodstuffs, these properties are in one way or another related to the temporal and spatial

\footnotetext{
${ }^{1}$ http://www.ni.com/labview/

${ }^{2}$ http://www.ecosimpro.com/
} 


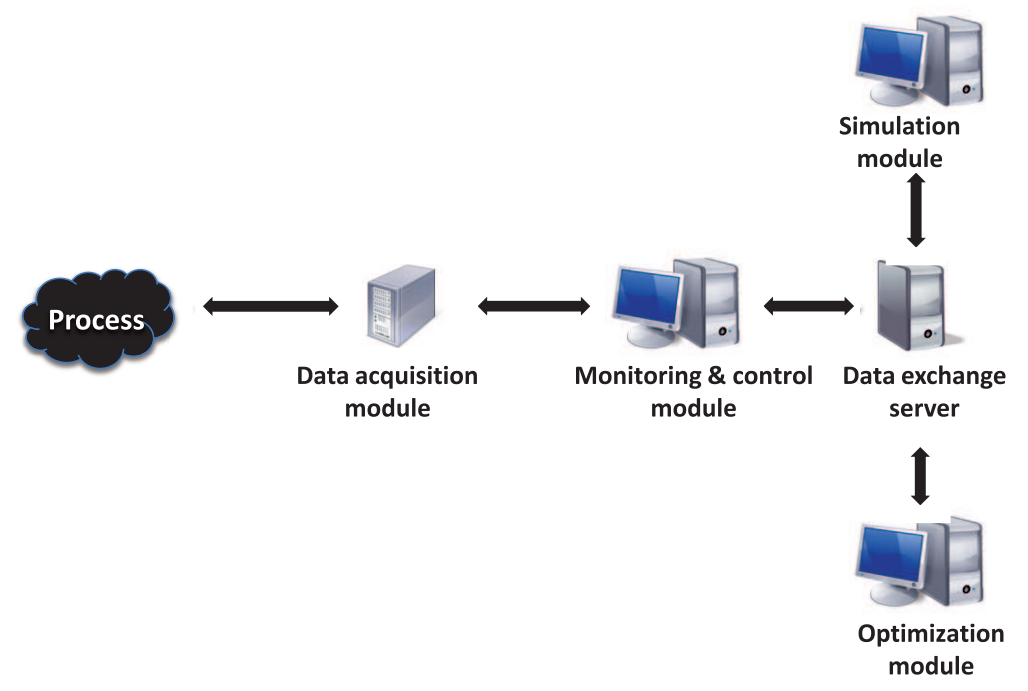

Figure 3: The software-hardware architecture. Information on the present status of the process is collected by the data acquisition module and sent to the plant simulator as initial conditions to predict future scenarios. The optimization module will interact with the simulator to compute the optimal policies. The resulting optimal profile will be sent back to the data acquisition as set-points.

distribution of temperature within the product, what calls for mathematical models described by partial differential equations (Balsa-Canto et al., 2002a).

The model employed to describe the evolution of retort temperature and pressure along the complete sterilization cycle is taken from Alonso et al. (1997). In order to make this work as self-contained as possible, a brief outline of the model, including its underlying mass and energy balances, is presented in Appendix A. Because in practice the system operates under well mixed conditions, the variables of interest are described by a set of ordinary differential equations, which formally can be expressed as:

$$
\dot{\mathbf{x}}=f(\mathbf{x} ; \theta)+g(\mathbf{x}, \mathbf{u} ; \theta),
$$

where $f(\mathbf{x} ; \theta)$ and $g(\mathbf{x}, \mathbf{u} ; \theta)$ are nonlinear vector fields of appropriate dimensions. $\mathbf{x}$ denotes the state vector with elements being the retort temperature and pressure, $T_{R}$ and $P_{R}$, respectively. The input vector $\mathbf{u}$ collects the relevant control variables, namely valve positions for the steam and air input streams as well as output streams such as drain and bleeder. Finally, $\theta$ denotes the vector of critical parameters associated to the process which, in our case, corresponds with the convective heat transfer coefficient of the vessel 
$h_{c}$, and steam and bleeder valve constants $\left(C_{v s}, \alpha_{s}\right)$ and $\left(C_{v b}, \alpha_{b}\right)$. Further information on valve parameters as well as the explicit relations underlying the formal representation (1), can be found in Appendix A.

The solid product to be sterilized is assumed to be packed in cylindrical RO-100 containers. In this way, temperature distribution in the product will be modeled by the so-called Fourier equation for heat conduction:

$$
\frac{\partial T_{\text {prod }}}{\partial t}=\alpha\left[\frac{\partial^{2} T_{\text {prod }}}{\partial z^{2}}+\frac{1}{r} \frac{\partial}{\partial r}\left(r \frac{\partial T_{\text {prod }}}{\partial r}\right)\right],
$$

where $r$ and $z$ are the cylindrical coordinates representing radius and length of the container, respectively (see Figure 4 for a representation of the spatial domain). $T_{\text {prod }}(r, z, t)$ stands for product temperature, and $\alpha$ for product thermal diffusivity.

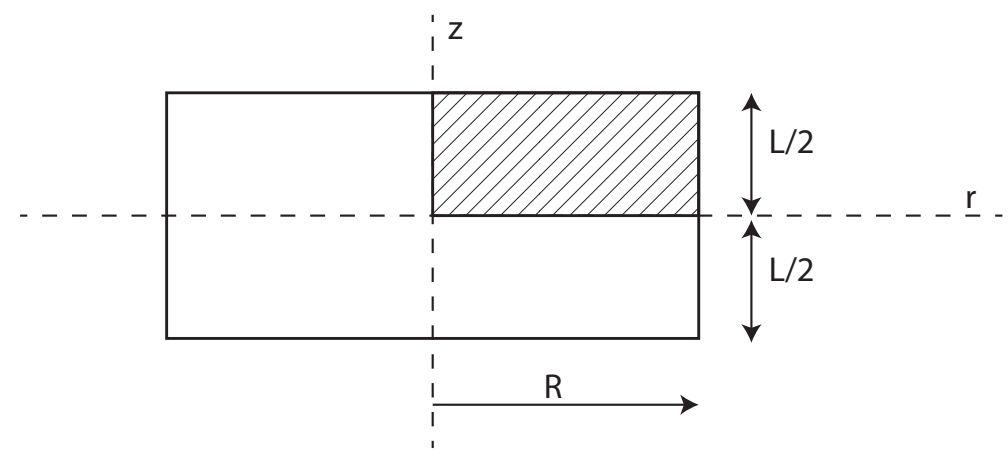

Figure 4: Section of a cylindrical can. By symmetry, Fourier equation needs to be solved only on a quadrant with coordinates taking values: $0 \leq r \leq R$ and $0 \leq z \leq L / 2$, where $R$ and $L$ are the total cylinder radius and length, respectively. For RO-100 containers, $R=0.0326 \mathrm{~m}$ and $L=0.03 \mathrm{~m}$.

The equation is completed with (Robin-type) boundary conditions of the form:

$$
\begin{aligned}
k\left(\frac{\partial T_{\text {prod }}}{\partial z}\right)_{z= \pm L} & =h\left(T_{R}(t)-T_{\text {prod }}(r, \pm L, t)\right) \\
k\left(\frac{\partial T_{\text {prod }}}{\partial r}\right)_{r=R} & =h\left(T_{R}(t)-T_{\text {prod }}(R, z, t)\right) \\
\left(\frac{\partial T_{\text {prod }}}{\partial r}\right)_{r=0}= & 0
\end{aligned}
$$


where $k$ is the thermal conductivity and $h$ represents the convective heat transfer coefficient between the product and the retort environment (saturated steam). These equations are combined with temperature dependent kinetics to account for the evolution of safety and quality parameters within the product. In testing the on-line optimizing control configuration, and following the works by Balsa-Canto et al. (2002a,b), lethality at the coldest point $F_{0}(t)$, and nutrient retention at the surface $\left(C_{i}^{s}\right)$ will be considered as the representative safety and quality parameters. Their time evolution at each location within the product will be modeled by the following ordinary differential equations:

$$
\begin{aligned}
\frac{d C_{i}^{s}}{d t} & =-\left(\frac{\ln 10}{D_{i, r e f}}\right) C_{i}^{s} \exp \left(\frac{T_{s}-T_{i, r e f}}{Z_{i, r e f}}\right), \\
\frac{d F_{0}}{d t} & =10^{\frac{T_{c}(t)-T_{M, r e f}}{Z_{M, r e f}}},
\end{aligned}
$$

where $T_{c}$ corresponds with temperature at the coldest point and $T_{s}$ surface temperature. Parameters $\left(Z_{M, r e f}, T_{M, r e f}\right)$ and $\left(Z_{i, r e f}, D_{i, r e f}, T_{i, r e f}\right)$ represent the kinetic coefficients for the target pathogen (subscript $M$ ) and the quality factor (subscript $i$ ). In our case and since we are interested in low-acid canned foods, the target pathogen parameters used to calculate commercial sterility will correspond with those of C. sporogenes ${ }^{3}$. On the other hand, thiamine retention will be the quality factor considered in this work. The list of physical and kinetic parameters used in this work is presented in Table 1.

Typical methods to numerically solve Eqns (2)-(5) make use of a certain spatial discretization scheme such as finite elements to approximate the original distributed system by a large set of ordinary differential equations, which is often computationally involved. This obstacle is particularly apparent when using the model in the context of a dynamic optimization problem where multiple simulations are needed for objective function evaluation (Balsa-Canto et al., 2002b). In order to overcome such limitation, reduced order dynamic representations with particular emphasis on the POD (Proper Orthogonal Decomposition) method have been employed in this work to capture the slow -thus representative- dynamics for temperature and quality.

\footnotetext{
${ }^{3}$ As it is well established in thermal processing, this is a microorganism very similar to $C$. botulinum but with a higher heat resistance. Thus, its destruction ensures that of $C$. botulinum spores.
} 
Table 1: The set of physical and kinetic parameter values employed in the model.

\begin{tabular}{lll}
\hline Parameter & Value & Units \\
\hline$\alpha$ & $1.11 \times 10^{-7}$ & $\mathrm{~m}^{2} \cdot \mathrm{s}^{-1}$ \\
$h$ & $10^{4}$ & $\mathrm{~W} \cdot \mathrm{m}^{-2} \cdot \mathrm{K}^{-1}$ \\
$k$ & 0.53 & $\mathrm{~W} \cdot \mathrm{m}^{-1} \cdot \mathrm{K}^{-1}$ \\
$D_{i, \text { ref }}$ & 10716 & $\mathrm{~s}$ \\
$z_{i, \text { ref }}$ & 25.5 & ${ }^{\circ} \mathrm{C}$ \\
$T_{i, \text { ref }}$ & 121.11 & ${ }^{\circ} \mathrm{C}$ \\
$z_{M, \text { ref }}$ & 10 & ${ }^{\circ} \mathrm{C}$ \\
$T_{M, r e f}$ & 121.11 & ${ }^{\circ} \mathrm{C}$ \\
\hline
\end{tabular}

Details on this particular method are presented in Appendix B (for the interested reader, see also Sirovich (1987); Balsa-Canto et al. (2002a); Garcia et al. (2007)).

\subsection{The optimal control configuration}

The control framework we propose in this work is presented in Figure 5 on block diagram form. It essentially consists of two interrelated layers: a regulatory feed-back control loop built around a set of robust tracking controllers, and a supervisory structure which is where optimal decisions are produced, based on the current state of the process (i.e current process and product temperatures, pressure, lethality, and product quality). This layer contains model calibration and predictive tools which make use of a virtual representation of the plant (the modelling/simulation block), combined with dynamic optimizers to explore future operation scenarios, which satisfying safety constraints, will ensure optimal final product quality. In what follows the different layers of the proposed control configuration will be discussed in detail.

\section{Model identification and calibration}

Identification and optimal experimental design methods are used to estimate the physical model parameters in Eqns (1) or (2) from the available measurements (Balsa-Canto et al., 2010; Balsa-Canto \& Banga, 2011). To that mission the process identification block of Figure 5 is devised, in a way to ensure that the uncertainty between the plant and the model is minimized or at least maintained under reasonable bounds during the operation.

Model identification is an activity which usually needs to be carried out 


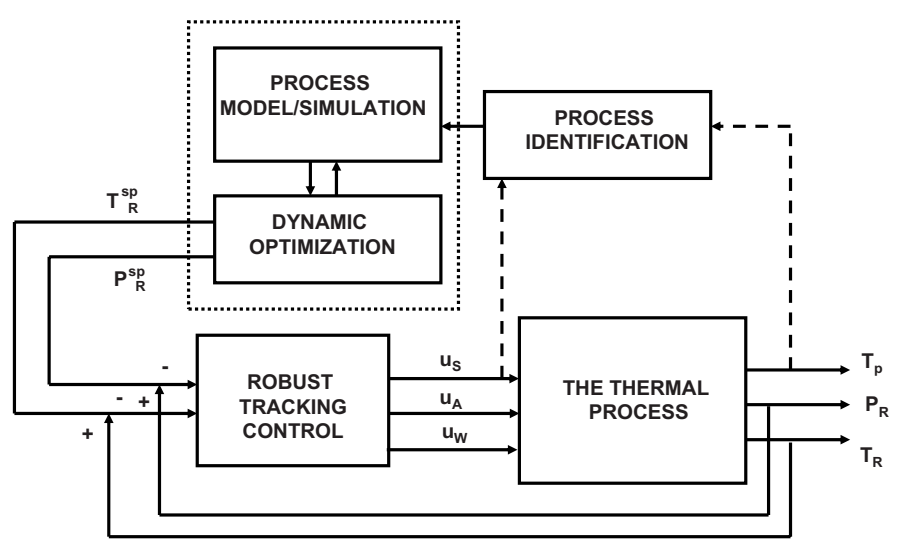

Figure 5: The block diagram depicts the complete control structure configuration for on-line optimal decision making in thermal processing, including the regulatory and supervisory control layers. The regulatory layer is composed of a robust controller (in our case a PI controller) that provides temperature and pressure set-point tracking. At the supervisory level, plant information is employed to calibrate the dynamic models of the plant (process identification) and to compute in the optimization block, optimal temperature and pressure set-point profiles to be sent to the regulatory controllers. $T_{R}$ and $P_{R}$ stand for retort temperature and pressure and $T_{p}$ for product temperature. $u_{s}, u_{a}$ and $u_{w}$ represent steam, air and water valve positions.

off-line. Model calibration however can be performed either on-line, during the process operation, or on a batch-to-batch basis, depending on the nature of the model or the estimation method selected. In this work, optimal experimental design and parameter estimation have been carried out on a batch-to-batch basis. Parameters to be estimated include valve related parameters (steam and bleeder valves as described in Appendix A and Table A.6) and convective heat transfer coefficient in the retort.

All computations have been performed using AMIGO (Advanced Model Identification using Global Optimization), a multi-platform toolbox developed by Balsa-Canto \& Banga (2011) which covers all the steps of the iterative identification procedure: local and global sensitivity analysis, multiexperiment parameter estimation, identifiability analysis and optimal experimental design. It also incorporates several state of the art simulators and local, global and hybrid nonlinear programming solvers.

\section{The regulatory layer}

Regulatory controllers with proportional and integral action (PI) are employed to track retort temperature and pressure set-points. During the heating stage, temperature is controlled by acting on the steam valve for fixed 
values of drain and bleeder (see Figure 2). On the other hand, a pressure PI controller acting on air is employed to end-up the heating stage and to initiate cooling by maintaining the retort under overpressure, and in this way to compensate for sudden pressure drops which could result into cracks or unsealing of containers.

Controllers have been designed under the Internal Model Control (IMC) paradigm (Ogunnaike \& Ray, 1994) assuming a first order open loop system's response in terms of temperature or pressure to steps in steam or air valve positions, respectively. The following structure has been proposed for both controllers:

$$
u(t)=\frac{\tau_{p}}{\lambda k_{p}} e(t)+\frac{1}{\lambda k_{p}} \int_{0}^{t} e(\xi) d \xi,
$$

where $e(t)=y^{s p}-y$ is the error between the set-points $y^{s p}$ and the current measurements $y$ (representing either $T_{R}$ or $P_{R}$ ). $k_{p}$ and $\tau_{p}$ are the gain and time constant associated to the input-output response, and $\lambda$ is the first order low-pass filter constant selected to tune the closed-loop response speed (Alonso et al., 1998). Equation (8) can be accommodated into the standard PI form, with $K$ and $\tau_{I}$ being the controller gain and integral time constant, by means of the following equivalences:

$$
K \equiv \frac{\tau_{p}}{\lambda k_{p}} \quad \text { and } \quad \frac{K}{\tau_{I}} \equiv \frac{1}{\lambda k_{p}} .
$$

In its final form, temperature and pressure controllers have been tuned so that $K=0.1$ and $\tau_{I}=20 \mathrm{~s}$. Typical open and closed loop retort responses in terms of temperature and pressure to set-points $T_{R}^{s p}$ and $P_{R}^{s p}$ are presented in Figures 6 and 7.

\section{Computing and implementing optimal control policies}

The dynamic optimization block, depicted in Figure 5 is the component responsible for taking optimal decisions at any time during the sterilization cycle, based on the present measurements and the estimated states of the process. To that purpose, this module is closely linked to the process simulator, which in fact takes the place of a virtual plant where future operation policies can be quickly examined in order to select the optimal one. The optimal control problems to solve will differ in the functional to be maximized or minimized. Nonetheless a safety constraint on the minimum acceptable lethality $F_{0}^{*}$ to be attained should be present in all formulations. Typical objectives may include the minimization of process time $t_{f}$ or the maximiza- 


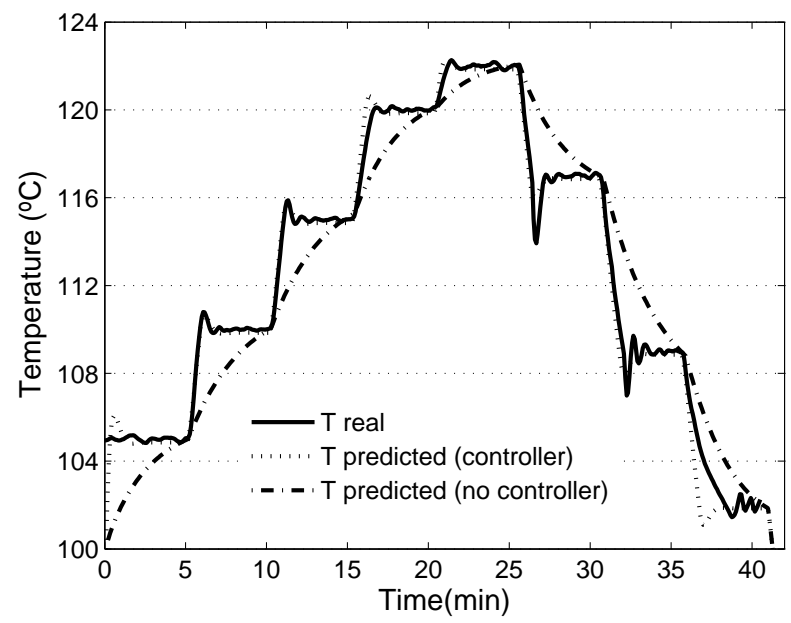

Figure 6: Evolution of retort temperature under PI control to a train of steps in the set point temperature. The corresponding open loop retort temperature evolution predicted by simulation is also represented for comparison purposes. Parameters for the PI controller (9) were $K=0.1, \tau_{I}=20 \mathrm{~s}$.

tion of a given quality factor (a nutrient retention for instance) compatible with a minimum acceptable lethality at a given final time $t_{f}^{*}$.

As it has been discussed in the introduction, such problems have been considered for long in the literature. However, most of them, if not all, reduced to finding an off-line optimal retort temperature profile without any consideration of the retort dynamics or unexpected disturbances that may render the computed profile suboptimal. As we will show in the next section, disregarding the dynamics of the retort and regulatory layer in the statement of the optimal control problem may lead more often than not to unfeasible retort temperature profiles, or to operations that are too energy demanding.

The optimal control problem we state next will explicitly consider the dynamics of the retort (1) and regulatory configuration (8), in addition to heat transfer in the product (2)-(5) and the dynamics for quality and lethality, modeled by Eqns (6) and (7). Subject to the above mentioned dynamic constraints, a typical optimal control problem can be formally stated as follows:

Optimal Quality Control. Find the retort temperature set-point profile $T_{R}^{s p}(t)$ along a sterilization cycle $0 \leq t \leq t_{f}^{*}$, which maximizes final nutrient retention $C_{i}^{s}\left(t_{f}^{*}\right)$ subject to a minimum acceptable lethality at the coldest point within the product i.e. $F_{0}\left(t_{f}^{*}\right) \geq F_{0}^{*}$, a retort temperature within a lower and 


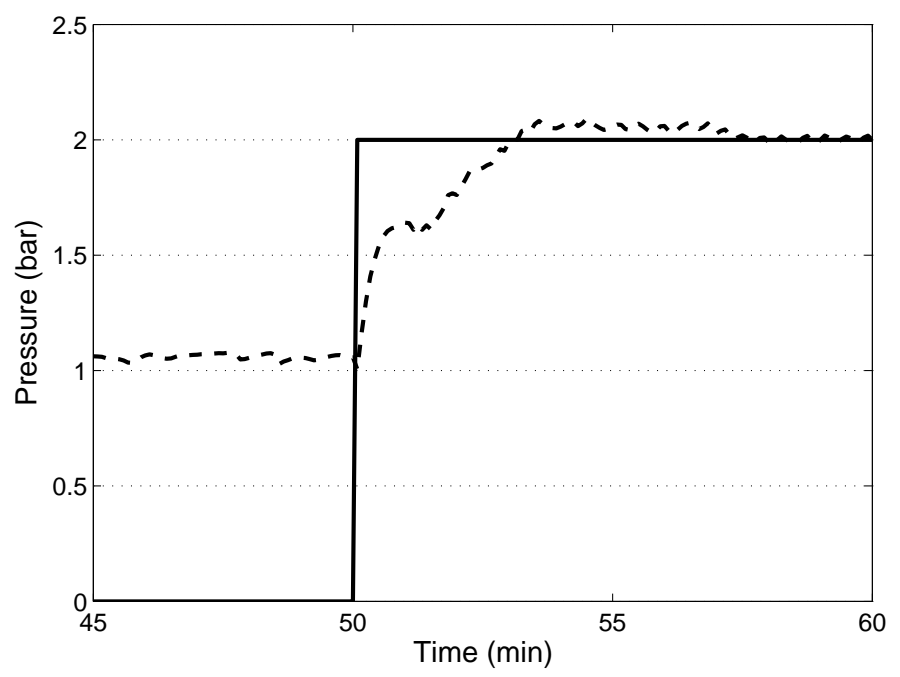

Figure 7: Pressure control tracking at the early cooling stage (pressure response is represented by the dashed line). Cooling water enters the retort producing a disturbance which is efficiently compensated by the controller. Parameters for the PI controller (9) were $K=0.1, \tau_{I}=20 s$.

upper bounds $\underline{T}_{R} \leq T_{R}(t) \leq \bar{T}_{R}$ and a final temperature at the coldest point $T_{c}\left(t_{f}^{*}\right) \leq T_{c}^{*}$. Note that when dealing with real time optimization, it may result convenient to include $t_{f}^{*}$ (final sterilization time) as a decision variable since it will add flexibility to the optimization problem, particularly at the last steps of the sterilization cycle.

In this work we will concentrate on the optimal quality control problem with the following specifications: $\underline{T}_{R}=102^{\circ} \mathrm{C}$ and $\bar{T}_{R}=125^{\circ} \mathrm{C}$. Maximum acceptable final temperature at the coldest point being $T_{c}^{*}=80^{\circ} \mathrm{C}$ and final lethality $F_{0}^{*}=8 \mathrm{~min}^{4}$. When $t_{f}$ is included as a decision variable in the optimization problem, lower and upper bounds become $\underline{t}_{f}^{*}=55$ and $\bar{t}_{f}^{*}=80$ minutes, respectively.

Numerically, the original optimal control problems are formulated as nonlinear programming problems (NLP) which approximate the original ones via the control vector parameterization (CVP) method. The resulting NLPs may then be solved with a global optimization solver -(see Banga et al., 2003) for details-. In this work a hybrid stochastic-deterministic method based on the

\footnotetext{
${ }^{4}$ This value corresponds with the commercial lethality for low acid prepackaged foods.
} 
scatter search approach has been employed. Details of the algorithm SSm (Scatter Search in matlab) can be seen in Egea et al. (2007). It is worth noting that since the dynamics of the retort is explicitly considered, the resulting retort temperature path is by construction feasible. Moreover, in order to ensure robustness, the computed policy will be sent to the regulatory layer, generally as a VRT (variable retort temperature) set-point. In the event of unexpected disturbances such as those in the steam supply, for instance, the optimal control problems can be recomputed and the new profiles implemented. This will prevent the over-processing of the product and in turn will save energy, ensure safety and maximize quality.

\section{Results and discussion}

\subsection{Model identification}

The model of the plant to be identified is that describing temperature and pressure evolution in the retort, which we formally represent by Eqn (1) and is described in Appendix A. These equations provide the boundary conditions associated to the model that predicts evolution of temperature, nutrient retention and lethality inside the food product (Eqns. (2)-(7)).

In this study, the goal of model identification was twofold: on the one hand to identify the functional dependency of steam and bleeder flows with respect to the control variables, namely valve openings. On the other hand, to identify unknown critical model parameters. The behavior of valves is usually represented by empirical relations obtained from experiments. In this work, the expressions employed are presented in Appendix A, Table A.6. Critical model parameters correspond to the convective heat transfer coefficient $h_{c}$ as well as valve characteristic parameters $C_{v s}, C_{v b}, \alpha_{s}$ and $\alpha_{b}$.

The characteristic valve parameters $C_{v j}$ are functions of the percentage of valve opening giving rise to linear, equal percentage or quick flow opening (Smith \& Corripio, 1997). The different possibilities have been collected into a battery of models which are presented in Appendix A. A model identification protocol has then been established to cover the following steps (Balsa-Canto et al., 2010):

- Structural identifiability ${ }^{5}$ analysis of all model candidates.

\footnotetext{
${ }^{5} \mathrm{~A}$ model is said to be structurally identifiable if parameters may be given unique values under ideal, noise free and continuous, experimental data.
} 
- Distinguishability ${ }^{6}$ analysis of all model candidates.

- Parameter estimation from experimental data. Experiments were performed in the pilot plant under different constant and time varying steam and bleeder valves opening profiles.

- Model discrimination and improvement of its predictive capabilities by means of model based optimal experimental design.

The values obtained for the critical parameters are presented in Table 2 . The performance of the resulting model is illustrated in Figure 8 where real and predicted temperature evolution in response to changes in the steam valve position are compared. As it can be seen in the figure there is a fair

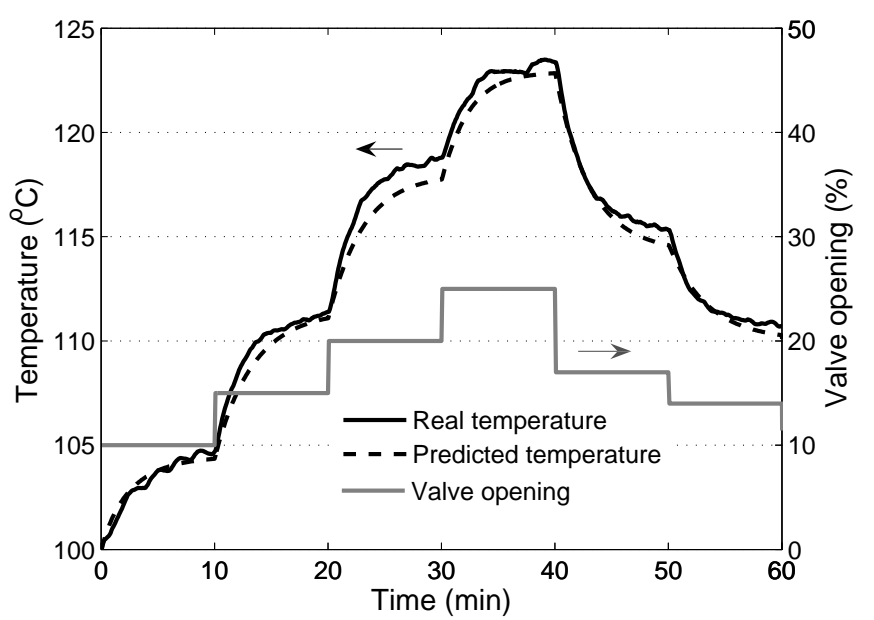

Figure 8: Experimental and predicted evolution of retort temperature in response to a train of steps in the steam valve. Bleeder opening is at $20 \%$, and drain opening at $55 \%$. 
Table 2: The resulting values for the critical parameters obtained after the iterative identification procedure.

\begin{tabular}{ccccc}
\hline$h_{c}\left(J / s K m^{2}\right)$ & $C_{v s}$ & $C_{v b}$ & $\alpha_{s}$ & $\alpha_{b}$ \\
\hline 11.983 & 5.2088 & 6.8834 & 1 & 1 \\
\hline
\end{tabular}

\subsection{Dynamic optimization}

In order to evaluate the effect of retort dynamics on the solution of the optimal quality control problem stated in Section 2.3, two optimal retort temperature set-point profiles $\left(T_{R}^{s p}(t)\right)$ have been computed: one of them, we will refer to as $O P 1$, considers only the dynamics of heat transfer within the product, defined by Eqn (2) with its associated boundary conditions. The other (OP2), in addition to equation (2) takes into account the dynamics of the retort described by Eqn (1).

The proper orthogonal decomposition method described in Appendix B has been used to produce fast simulations of the product dynamics. For the case under study five basis functions, what amounts to solving 5 ODEs (ordinary differential equations), were enough to provide a satisfactory accuracy. Note that in contrast, a classical method such as the finite element method results into a system with more than 300 ODEs, i.e., with around 60 times more equations than with the POD method.

Following the CVP paradigm, the optimal profiles $T_{R}^{s p}(t)$ are found within the set of step-wise continuous functions. For illustrative purposes, profiles consisting of 4 steps with 12.5 minutes length each, were considered to compare $O P 1$ and $O P 2$ cases $^{7}$. Results for OP1 and OP2 in terms of final retention, lethality and final temperature at the center of the product are presented in Table 3. As it can be seen from the results, both solutions respect constraints on final lethality $\left(F_{0}^{*}=8\right.$ minutes) and maximum allowed temperature at the center of the product $\left(T_{c}^{*}=80^{\circ} \mathrm{C}\right)$ with a very similar value of the objective function. However, the implementation in pilot plant of the temperature profile corresponding to $O P 1$ leads to a final lethality $F_{0}=7.53$ minutes, clearly below the constraint. This shows that disregard-

\footnotetext{
${ }^{7}$ In this case the final sterilization time $t_{f}^{*}$ is not included as a decision variable since the objective of this experiment is to show the impact of disregarding the retort dynamics on the safety parameter.
} 
Table 3: Comparison of results for $O P 1$ (without retort dynamics) and $O P 2$ (with retort dynamics). Computed values correspond with the solution of the optimal control problem while experimental values are those obtained after implementation in pilot plant.

\begin{tabular}{lccccc}
\hline & \multicolumn{2}{c}{$O P 1$} & & \multicolumn{2}{c}{$O P 2$} \\
\cline { 2 - 3 } \cline { 5 - 6 } & Computed & Experimental & & Computed & Experimental \\
\hline Retention (\%) & 67.07 & 67.63 & & 67.14 & 66.97 \\
Lethality (min) & 8.00 & 7.53 & & 8.00 & 8.01 \\
$T_{c}\left({ }^{\circ} \mathrm{C}\right)$ & 79.97 & 79.51 & & 79.98 & 79.76 \\
\hline
\end{tabular}

ing the dynamics of the process (the retort) may lead to unfeasible solutions, i.e. solutions that do not verify the safety constraint (in our case $F_{0} \geq 8$ minutes).

The optimal profile associated to $O P 2$ is depicted in Figure 9 together with the experimental evolution of retort temperature, and the temperature at the center of the product (both predicted and experimental). The evolution of the retention and lethality obtained by the set-point profiles corresponding to $O P 1$ and $O P 2$ are presented in Figure 10.

Note that unfeasible solutions in $O P 1$ could be avoided by explicitly constraining the retort temperature profile. Unfortunately it is not trivial to link constraints in the profile, for example maximum temperature rate change, with the actual manipulated variables (valve position, for instance). In addition such relationships might depend on the actual operation due to the intrinsic nonlinearity of the process unit. Safe values can be defined but they are likely to result into an unnecessary slow process with detrimental effects in quality or operation parameters (e.g. slower processes will lead to longer process times). In summary, unfeasible solutions can be avoided by constraining the temperature profile but that will lead in most cases to suboptimal operation. A precise characterization of limits on the achievable profiles must rely either on extensive experiments on the plant (energy and time consuming) or on the use of a detailed model of the plant. This second approach is the one we propose, although not to be implemented on a trial and error basis, but by letting the optimizer to implicitly find the feasible limits on the temperature profiles that optimize the selected criteria. 


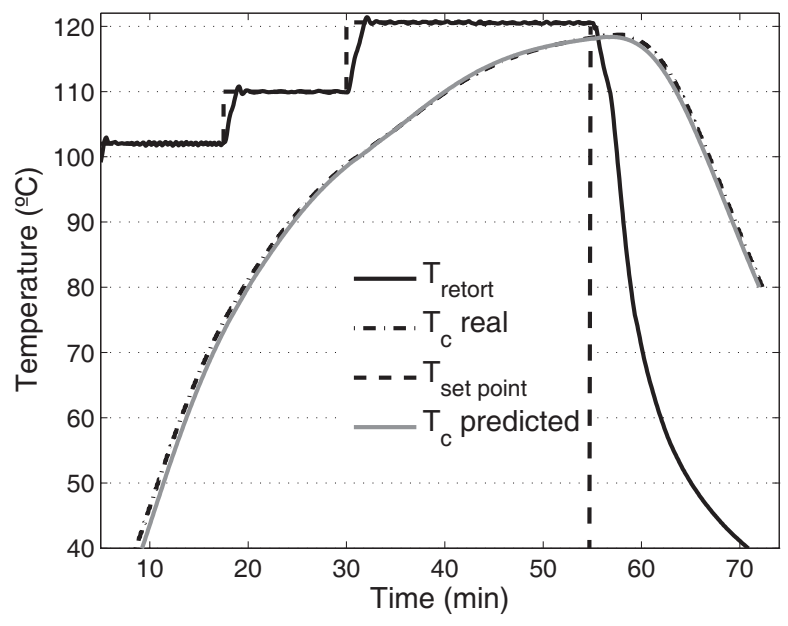

Figure 9: Implementation of the optimal retort temperature profile computed by considering the dynamics of the product and the process. Cooling stage starts at the dashed vertical line around 55 minutes. From that time on, temperature set-point coincides with cooling water temperature.

\subsection{Real time optimization}

In the event of process disturbances that might cause deviations from the prescribed safety or quality constraints, the optimal quality control problem must be recomputed based on the present available information of the system. This has been done by means of a real time optimization scheme, implemented as follows:

1. Given the current state of the process (i.e. retort and product temperature as well as actual product lethality and retention) an optimal control problem is solved.

2. The resulting optimal retort temperature profile is implemented in the form of set-points on the regulatory layer

3. Periodically, predictions are compared with the real state of the plant to check for possible deviations.

4. If any significant deviation occurs a new optimal profile is recomputed and implemented (steps 1 and 2).

In order to solve for the first time the optimal control problem, a careful analysis of the effect of varying the number of steps in the control vector 


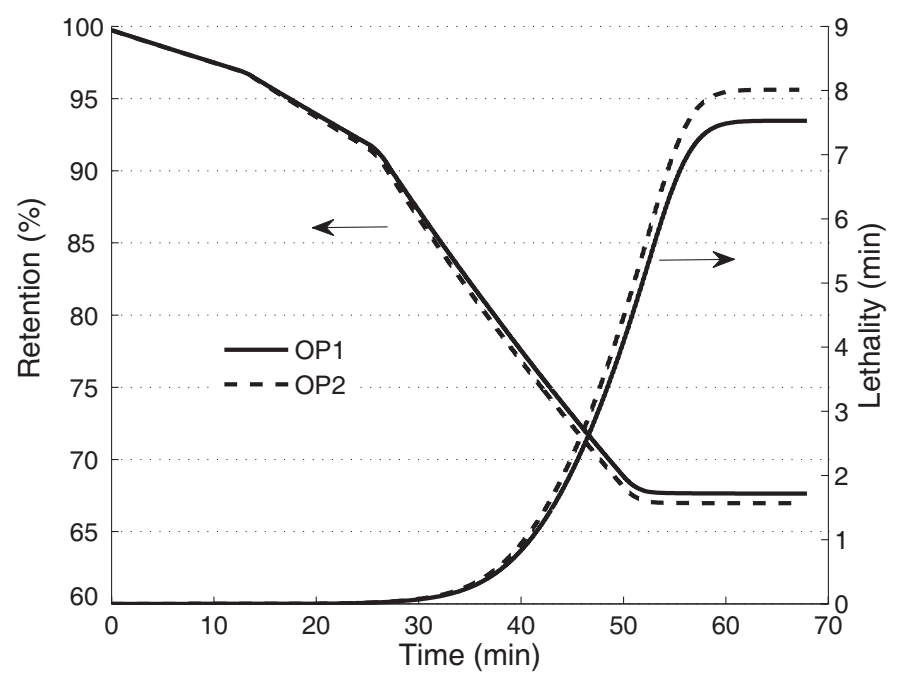

Figure 10: Evolution of retention and lethality corresponding to the implementation of the optimal quality control profiles considering the dynamics of the product (OP1) and the dynamics of the product and the process (OP2).

parameterization approach was performed. To do so the optimization problem was solved by means of SSm global optimizer (Egea et al., 2007), using an increasing number of steps. The results, presented in Table 4 show that the use of variable retort profiles (VRT) offer a substantial improvement in nutrient retention with respect to the widely used constant retort profiles (CRT), as already shown by Teixeira et al. (1975), Banga et al. (1991) or Durance (1997) . In addition, the results suggest that 8 control steps seem to be enough since larger discretization levels do not lead to relevant improvements in the maximum retention. It should be noted that the corresponding number of decision variables and the associated computational cost are also reasonable for the purpose of real time optimization. In this case, the final sterilization time $t_{f}$ is included as a decision variable in order to add flexibility to the optimization problem.

The CPU time shown in the table could be considered as a reference of the maximum CPU time required for on-line optimization, because global optimizers will only be used in the case of large perturbations. In addition it should be noted that typical on-line optimization (with local methods) takes no more than a few seconds and the computational cost decreases as the process evolves since the number of control elements is being reduced accordingly. 
Table 4: Comparison in terms of maximum retention reached, between different control discretization schemes. A CRT (constant retort temperature) profile corresponds with one step. Number of control steps larger than 8 did not result into significant improvements on the objective function.

\begin{tabular}{ccc}
\hline Number of steps & Optimal retention (\%) & CPU Time (s) \\
\hline 1 & 63.461 & 17 \\
2 & 66.654 & 28 \\
4 & 67.166 & 53 \\
6 & 67.585 & 175 \\
8 & 67.771 & 219 \\
10 & 67.774 & 376 \\
\hline
\end{tabular}

In the case of small perturbations, a local direct search optimization solver is used. Note that the optimal solution will be close to the original thus a local optimizer offers a good compromise between convergence rate and computational cost. In the event of larger perturbations, it may happen that feasibility of the solution may not be guaranteed or that the new optimum is far from the previous one. In these scenarios a global optimization method SSm (Egea et al., 2007) is used to prevent convergence to suboptimal solutions. Note, in addition, that the processing time may increase depending on the magnitude and location of the perturbation.

The recomputed optimal profiles are implemented in the plant as soon as they become available and that depends on the time for the optimizer to obtain a solution. As discussed above, the CPU times in our case ranged from seconds to a few minutes.

In order to test the performance of the proposed real-time optimal control strategy, a sudden pressure drop in the steam supply was induced during the process. Such scenario is typical of industrial plants where many retorts are simultaneously operating what results into extremely large steam demands which may cause retort pressure and temperature to fall down. This situation is illustrated in Figure 11 where the process is being affected by a pressure drop that starts at 37.7 minutes and lasts for 50 seconds.

Figure 12 represents both the optimal temperature set-point profile computed off-line and the one recomputed on-line. The corresponding evolution of retention and lethality are depicted in Figure 13. Its final values together with temperature at the center of the product are summarized in Table 5 . 


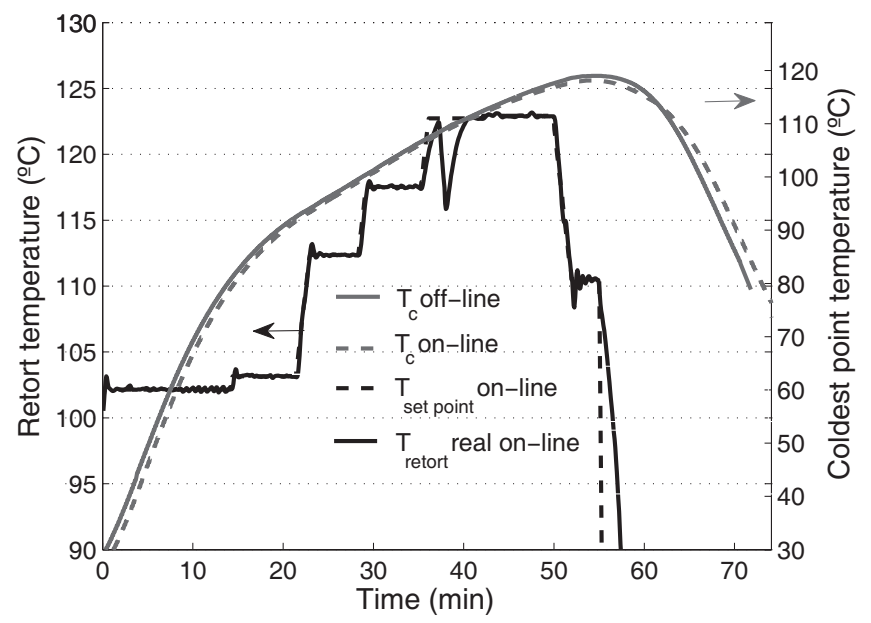

Figure 11: On-line implementation of the optimal retort temperature profile in the event of disturbances. Disturbance (a pressure drop that last for 50 seconds) occurs at 37.7 minutes. A new optimal temperature set point profile is recomputed to keep specifications.

Table 5: Results of real time optimization in the presence of disturbances.

\begin{tabular}{lcc}
\hline & Off line implementation & On-line implementation \\
\hline Retention (\%) & 68.5 & 67.0 \\
Lethality (min) & 7.21 & 8.47 \\
$T_{c}\left({ }^{\circ} \mathrm{C}\right)$ & 80.0 & 80.0 \\
\hline
\end{tabular}

As illustrated by the figures, on-line re-optimization during the sterilization cycle guarantees safety specifications while minimizing over-processing. In fact, the optimization scheme corrects the pressure drop by increasing the set point temperatures in the remaining steps compensating the sharp drop of temperature due to the pressure failure.

\section{Conclusions}

This work presents the development and validation of a real-time optimization architecture to operate thermal sterilization of packaged foods in batch retorts so as to maximize nutrient retention while satisfying safety 


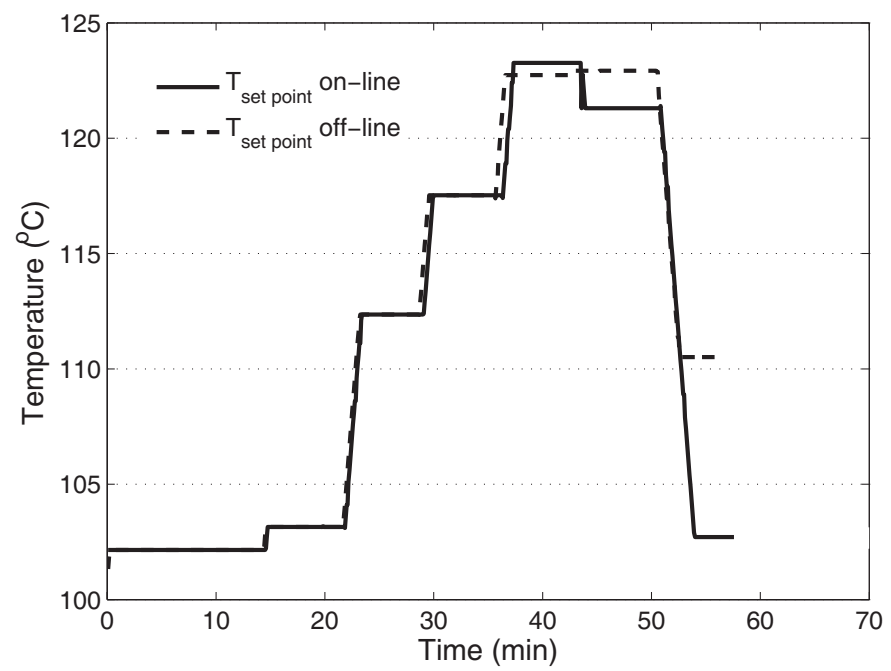

Figure 12: A comparison of the optimal temperature set-points computed off-line and online in the event of disturbances. The temperature set-point profile has been discretized in 8 elements of 7 minutes length each.

related constraints under retort temperature deviations due to unexpected process disturbances.

The proposed architecture consists of two interrelated layers: a regulatory feed-back loop built around the robust tracking controller, and the supervisory structure which is where optimal decisions are taken based on the current state of the process. This supervisory structure relies on the synchronous combination of reliable and computationally efficient models of the process and food product with a efficient and robust optimization structure. In this regard, first principle based models were identified and validated to describe temperature and pressure within the retort and temperature, lethality and nutrient retention within the food product. Computationally efficient versions of these models were derived by means of model reduction techniques based on POD expansion. Finally, advanced dynamic optimization techniques, that make use of global hybrid optimization methods, were used to compute off-line and on-line optimal operation conditions.

As a proof of concept the methodology has been applied to the sterilization of solid foodstuffs. However the methodology by no means restricts to homogeneous products. Inhomogeneous solids as well as other geometries or packages can be accommodated into the same paradigm. In terms of modeling, the finite element method is general enough to handle a variety of 


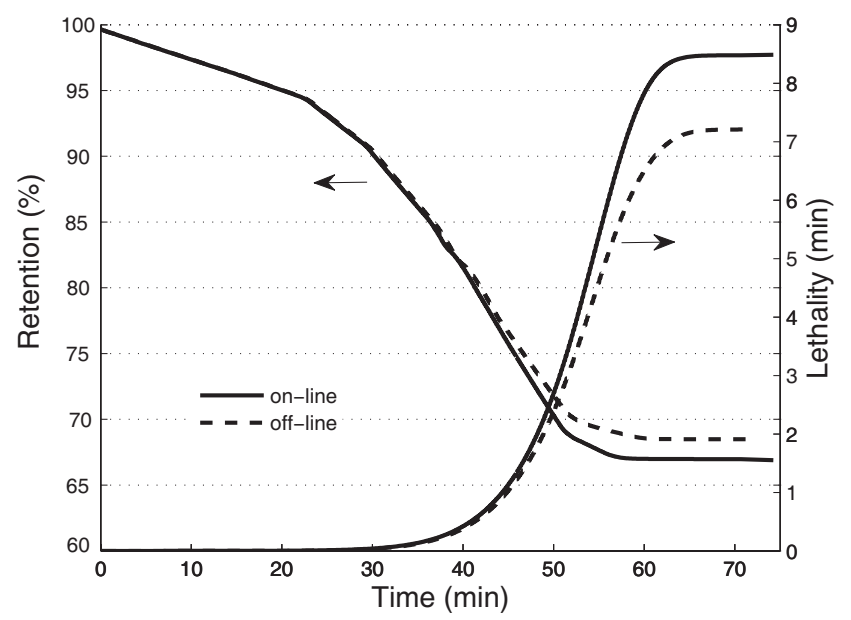

Figure 13: Evolution of retention and lethality corresponding to the implementation of the on-line and off-line optimal temperature profiles.

geometries or diverse non-homogeneous transfer mechanisms including natural convection, for instance. Of course, higher complexity would increase the computational burden which in turns could jeopardize the real time specification. Nonetheless, as it has been discussed previously, methods such as PODs or spectral decomposition provide accurate low dimensional approximations that can be successfully employed in real time applications.

The performance of the proposed RTO architecture was validated experimentally on a pilot plant located at IIM-CSIC, testing it under process perturbations and showing how it is possible to optimally command the system to attain quality specifications while satisfying safety related constraints.

\section{Acknowledgements}

This work has been funded by the Spanish Ministry of Science and Innovation (SMART-QC, AGL2008-05267-C03-01), Xunta de Galicia (IDECOP, 08DPI007402PR) and FP7 CAFE project (KBBE-2007-1-212754). Ana I Arias Méndez acknowledges financial support from the CSIC JAE-predoc programme. 


\section{References}

Abakarov, A., Sushkov, Y., Almonacid, S., \& Simpson, R. (2009). Multiobjective optimization approach: thermal food processing. Journal of Food Science, 74, E471-E487.

Akterian, A. (1999). On-line control strategy for compensating for arbitrary deviations in heating medium temperature during batch thermal sterilization processes. Journal of Food Engineering, 39, 1-7.

Alonso, A. A., Banga, J. R., \& Perez-Martin, R. I. (1997). A complete dynamic model for the thermal processing of bioproducts in batch units and its application to controller design. Chemical Engineering Science, 52, 1307-1322.

Alonso, A. A., Banga, J. R., \& Perez-Martin, R. I. (1998). Modelling and adaptive control of a batch sterilization process. Computers $\& 5$ Chemical Engineering, 22, 445-458.

Ansorena, M. R., \& Salvadori, V. O. (2011). Optimization of thermal processing of canned mussels. Food Science and Technology International, 17, 449-458.

Balsa-Canto, E., Alonso, A. A., \& Banga, J. R. (2002a). A novel, efficient and reliable method for thermal process design and optimization. Part I: theory. Journal of Food Engineering, 52, 227-234.

Balsa-Canto, E., Alonso, A. A., \& Banga, J. R. (2010). An iterative identification procedure for dynamic modelling of biochemical networks. $B M C$ Systems Biology, 4, 1-18.

Balsa-Canto, E., \& Banga, J. R. (2011). Amigo, a toolbox for advanced model identification in systems biology using global optimization. Bioinformatics, 27, 2311-2313.

Balsa-Canto, E., Banga, J. R., \& Alonso, A. A. (2002b). A novel, efficient and reliable method for thermal process design and optimization. Part II: applications. Journal of Food Engineering, 52, 235-247.

Banga, J. R., Balsa-Canto, E., \& Alonso, A. A. (2008). Quality and safety models and optimization as part of computer-integrated manufacturing. Comprehensive Reviews in Food Science and Food Safety, 7, 168-174. 
Banga, J. R., Balsa-Canto, E., Moles, C. G., \& Alonso, A. A. (2003). Improving food processing using modern optimization methods. Trends in Food Science and Technology, 14, 131-144.

Banga, J. R., Perez-Martin, R. I., Gallardo, J. M., \& Casares, J. J. (1991). Optimization of thermal processing of conduction-heated canned foods, study of several objective functions. Journal of Food Engineering, 14, $25-51$.

Camacho, E., \& Bordóns, C. (1995). Model predictive control in the process industry. London: Springer-Verlag.

Chalabi, Z. S., van Willigenburg, L. G., \& van Straten, G. (1999). Robust optimal receding horizon control of the thermal sterilization of canned foods. Journal of Food Engineering, 40, 207-218.

Chen, C. R., \& Ramaswamy, H. S. (2002). Modeling and optimization of variable retort temperature (VRT) thermal processing using coupled neural networks and genetic algorithms. Journal of Food Engineering, 53, 209220 .

Chen, G., Campanella, O. H., Corvalan, C. M., \& Haley, T. A. (2008). Online correction of process temperature deviations in continuous retorts. Journal of Food Engineering, 84, 258-269.

Durance, T. D. (1997). Improving canned food quality with variable retort temperature processes. Trends in Food Science \& Technology, 8, 113-118.

Egea, J. A., Rodriguez-Fernandez, M., Banga, J. R., \& Marti, R. (2007). Scatter search for chemical and bioprocess optimization. Global Optimization, 37, 481-503.

Enitan, A. M., \& Adeyemo, J. (2011). Food processing optimization using evolutionary algorithms. African Journal of Biotechnology, 10, 1612016127.

Erdogdu, F., \& Balaban, M. O. (2003). Complex method for nonlinear constrained multi-criteria (multi-objective function) optimization of thermal processing. Journal of Food Process Engineering, 26, 357-375. 
Flores-Cerrillo, J., \& MacGregor, J. F. (2005). Latent variable mpc for trajectory tracking in batch processes. Journal of Process Control, 15, 651-663.

Garcia, M. R., Vilas, C., Banga, J. R., \& Alonso, A. A. (2007). Optimal field reconstruction of distributed process systems from partial measurements. Industrial \&6 Engineering Chemistry Research, 46, 530-539.

Koribilli, N., Aravanudan, K., \& Varadhan, M. U. S. V. A. (2011). Quantifying enhancement in heat transfer due to natural convection during canned food thermal sterilization in a still retort. Food and Bioprocess Technology, 4, 429-450.

Kurtanjek, Z. (2008). Opportunities and challenges of model predictive control in food technologies. In Proceeding of the 4 th Central European Congress of Food, Biotechnologists and Nutritionists. Vol 1. (pp. 105-110).

Lopez, A. (1987). A complete course in canning. Baltimore, 12th Ed: Canning Trade Inc.

Martins, R. C., Lopes, V. V., Vicente, A. A., \& Teixeira, J. A. (2008). Computational shelf-life dating, complex systems approaches to food quality and safety. Food and Bioprocess Technology, 1, 207-222.

Miri, T., Tsoukalas, A., Bakalis, S., Pistikopoulos, E. N., Rustem, B., \& Fryer, P. J. (2008). Global optimization of process conditions in batch thermal sterilization of food. Food and Bioprocess Technology, 87, 485494.

Nadkarni, M. M., \& Hatton, T. A. (1985). Optimal nutrient retention during the thermal processing of conduction-heated canned foods, applications of the distributed minimum principle. Journal of Food Science, 50, 13121321.

Ogunnaike, B. A., \& Ray, W. H. (1994). Process dynamics, modelling and control. New York: Oxford University Press.

Ravindran, S. S. (2000). A reduced-order approach for optimal control of fluids using proper orthogonal decomposition. International Journal for Numerical Methods in Fluids, 34, 425-448. 
Saguy, I., \& Karel, M. (1979). Optimal retort temperature profile in optimizing thiamine retention in conduction-type heating of canned foods. Journal of Food Science, 44, 11485-11490.

Sendin, J. O. H., Alonso, A. A., \& Banga, J. R. (2010). Efficient and robust multi-objective optimization of food processing: A novel approach with application to thermal sterilization. Journal of Food Engineering, 98, 317324.

Simpson, R., \& Abakarov, A. (2011). Optimization of food thermal processing: Sterilization stage and plant production scheduling. In Food Engineering Interfaces Food Engineering Series (pp. 261-284). Springer New, York.

Simpson, R., Abakarov, A., \& Teixeira, A. (2008). Variable retort temperature optimization using adaptive random search techniques. Food Control, $19,1023-1032$.

Simpson, R., Almonacid, S., \& Mitchell, M. (2004). Mathematical model development, experimental validation and process optimization, retortable pouches packed with seafood in cone frustum shape. Journal of Food Engineering, 63, 153-162.

Simpson, R., Figueroa, I., \& Teixeira, A. (2007a). Simple, practical and efficient on-line correction of process deviations in batch retort through simulation. Food Control, 18, 458-465.

Simpson, R., Teixeira, A., \& Almonacid, S. (2007b). Advances with intelligent on-line retort control and automation in thermal processing of canned foods. Food Control, 18, 821-833.

Sirovich, L. (1987). Turbulence and the dynamics of coherent structures. Part I: coherent structures. Quaterly of Applied Mathematics, 45, 561-571.

Smith, C. A., \& Corripio, A. B. (1997). Principles and practice of automatic process control. New York, 2nd Ed: Wiley.

Teixeira, A. A., \& Tucker, G. S. (1997). On-line retort control in thermal sterilization of canned foods. Food Control, 8, 13-20. 
Teixeira, A. A., Zinsmeister, G. E., \& Zahradnik, J. W. (1975). Computer simulation of variable retort control and container geometry as possible means of improving thiamine retention in thermally processed foods. Journal of Food Science, 40, 656-659.

\section{Appendix A. Mathematical Model of the Thermal Processing Unit}

A complete mathematical description of thermal processing in batch retorts that includes all stages of the sterilization cycle can be found in Alonso et al. (1997). The model is based on mass and energy balances for liquid water, steam and air on the retort under well mixed conditions and the ideal gas assumption. For the sake of completeness, a summary of the main equations and constitutive relations employed to describe heat and mass flows is presented next.

Using the subscripts $s, w$ and $a$, to denote steam, water and air, respectively, mass balances in the retort take the form:

$$
\begin{aligned}
\frac{d m_{s}}{d t} & =F_{s}^{i}-x_{s} F_{b}+\gamma, \\
\frac{d m_{w}}{d t} & =F_{w}^{i}-F_{d}-\gamma, \\
\frac{d m_{a}}{d t} & =F_{a}^{i}-x_{a} F_{b},
\end{aligned}
$$

where $F$ represents flows, superscript $i$ input streams, and $x_{s}, x_{a}$, are the mass fractions of steam and air in the bleeder stream. The expressions that describe flows through steam and bleeder valves during the heating stage as a function of the states (temperature, pressure), steam pressure supply $\left(P_{\text {supply }}\right)$ and valve positions have been taken from Smith \& Corripio (1997) and are presented in Table A.6.

The term $\gamma$ in the right hand side of (A.1) or (A.2) represents the vapor flow transferred from the liquid phase to the vapor phase within the retort. This term was first introduced in Alonso et al. (1997) to describe scenarios in which both liquid and vapor phases coexist as it is the case during the early stages of cooling or under incomplete drainage during heating. At every instant, $\gamma$ must be such that it balances the mass of steam accumulated in the available volume with that in equilibrium at the retort temperature. Thus it can be computed as the value which makes zero the following equation:

$$
\Psi(\gamma)=m_{s}(\gamma)-\frac{P^{e q}\left(T_{R}\right) V_{s}(\gamma) M_{w}}{R T_{R}},
$$


where $P^{e q}$ is the vapor pressure of water related to the retort temperature by Antoine's law ${ }^{8} . M_{w}$ denotes molecular weight of water (in $\mathrm{kg} / \mathrm{mol}$ ) and $R$ the universal constant of gases $(R=8.314 \mathrm{~J} / \mathrm{mol} K)$. Finally, $V_{s}$ in (A.4) represents the volume available to the vapor phase, which is obtained as the difference between that of the retort $V_{R}$ and the one occupied by the liquid water phase (density $\rho_{w}$ ), e.g.

$$
V_{s}=V_{R}-\frac{m_{w}(\gamma)}{\rho_{w}} .
$$

It must be noted that during the heating stage all the condensate formed is drained, thus $\gamma=0$ what simplifies the mass balances.

Under the same assumptions, the balance for internal energy $U$ in the retort is of the form:

$$
\frac{d U}{d t}=\sum_{j} F_{j}^{i} \mathcal{H}_{j}^{i}-F_{b}\left(\mathcal{H}_{s}^{b} x_{s}+\mathcal{H}_{a}^{b} x_{a}\right)-F_{d} \mathcal{H}_{w}^{d}-Q_{t o t},
$$

where:

$$
U=\sum_{j} m_{j} \mathcal{U}_{j} .
$$

$\mathcal{U}_{j}$ represents internal energy per unit of mass, and the summations in (A.6) and (A.7) extend to all components/phases (i.e. $s, w$ and $a$ ) in their respective input streams or in the retort. $\mathcal{H}_{s}^{b}, \mathcal{H}_{w}^{d}$ and $\mathcal{H}_{a}^{b}$ denote enthalpy per unit of mass for saturated steam, water and air at the conditions of the bleeder and drain streams, as indicated by the superscript. Finally, the term $Q_{t o t}$ represents the heat transfer rate associated to all relevant heat sinks. These include the heat absorbed by the retort itself $\left(Q_{\text {ret }}\right)$, the product $Q_{\text {prod }}$, and the heat released to the environment by radiation and convection $\left(Q_{\text {rad }}\right.$ and $\left.Q_{\text {conv }}\right)$, so that:

$$
Q_{\text {tot }}=Q_{\text {ret }}+Q_{\text {prod }}+Q_{\text {rad }}+Q_{\text {conv }} .
$$

The expressions employed for the different heat transfer rates are presented in Table A.7. During the heating stage, model (A.1)-(A.6) simplifies since only saturated steam is present in the retort. In this case $U$ (relation (A.7))

\footnotetext{
${ }^{8} \mathrm{As}$ it can be found in any standard textbook on process thermodynamics, the expression reads $P^{e q}=\exp \left(A+\frac{B}{T-C}\right)$, with $A, B$ and $C$ being parameters dependent on the particular chemical species, water in this case.
} 
Table A.6: Expressions for flow through valves as described in Smith \& Corripio (1997) with characteristic valve parameter $C_{f}=0.9$ and $\alpha_{j}$ identifying the type of valve nonlinearity. Valve opening $u_{j}$ accepts values between 0 and $1 . P_{\text {supply }}$ defines the pressure at which steam is supplied to the unit and $P_{a t m}$ is atmospheric pressure.

$$
\begin{aligned}
& F_{s}^{i}=3.4 \cdot 10^{-8} \delta_{s}\left(u_{s}\right) C_{f} P_{r e d} \sqrt{G_{f}\left(w_{s}-0.148 w_{s}^{3}\right)} \\
& F_{b}=3.4 \cdot 10^{-8} \delta_{b}\left(u_{b}\right) C_{f} P^{e q} \sqrt{G_{f}\left(w_{b}-0.148 w_{b}^{3}\right)} \\
& w_{s}=\frac{1.63}{C_{f}} \sqrt{\frac{P_{\text {supply }}-P_{R}}{P_{\text {supply }}}} \\
& w_{b}=\frac{1.63}{C_{f}} \sqrt{\frac{P_{R}-P_{a t m}}{P_{R}}} \\
& \delta_{j}\left(u_{j}\right)=C_{v j} u_{j}^{1 / \alpha_{j}} \text { with } j \text { being either steam }(s) \text { or bleeder }(b) \text { valves. }
\end{aligned}
$$

Table A.7: Heat transfer rates associated to the main sinks. $m_{\text {prod }}$ and $C_{p_{\text {prod }}}$ are the total mass and heat capacity of the product. $m_{r e t}, A_{\text {ret }}$ and $C_{p_{\text {ret }}}$ are the total retort mass, area and heat capacity, respectively. $\varepsilon=0.99$ is the emissivity of the object, and $\sigma=5.6710^{-8} \mathrm{~W} / \mathrm{m}^{2} \mathrm{~K}^{4}$ is the Stefan-Boltzmann constant.

$$
\begin{aligned}
& Q_{\text {ret }}=m_{\text {ret }} C_{p_{\text {ret }}} \frac{d T_{R}}{d t} \\
& Q_{\text {prod }}=m_{\text {prod }} C_{p_{\text {prod }}} \int_{V} \dot{T}_{\text {prod }} d \xi \\
& Q_{\text {rad }}=\varepsilon \sigma A_{\text {ret }}\left(T_{R}^{4}-T_{\text {ext }}^{4}\right) \\
& Q_{\text {conv }}=h_{c} A_{\text {ret }}\left(T_{R}-T_{\text {ext }}\right)
\end{aligned}
$$


reduces to $U=m_{s}\left(T_{R}\right) \mathcal{U}_{s}\left(T_{R}\right)$. Computing its time derivative, substituting Eqns (A.1) and (A.6) into the resulting expression and reordering terms, we end up with the following nonlinear differential equation:

$$
\beta \frac{d T_{R}}{d t}=F_{s}-F_{b}-\frac{Q_{r a d}+Q_{c o n v}}{\lambda},
$$

with $\beta$ of the form:

$$
\beta=\left[\frac{m_{r e t} C_{p r e t}}{\lambda}-\left(\frac{B T}{(T-C)^{2}}+1\right) \frac{V_{s} P^{e q}}{R T_{R}^{2}}\right],
$$

which describes the evolution of the retort temperature as a function of steam and bleeder flows.

\section{Appendix B. The proper orthogonal decomposition}

Classical numerical methods for solving partial differential equations of the form (2) may result unsuitable for real time tasks like optimization. In this section we present an efficient order reduction technique known as the proper orthogonal decomposition (POD) (Sirovich, 1987) to overcome this limitation. For the sake of clarity, let us re-write Eqn (2) as follows:

$$
\frac{\partial T_{\text {prod }}}{\partial t}=\alpha \mathcal{A} T_{\text {prod }}
$$

where $\mathcal{A}$ represents the laplacian operator in cylindrical coordinates

$$
\mathcal{A}=\frac{\partial^{2}}{\partial z^{2}}+\frac{1}{r} \frac{\partial}{\partial r}\left(r \frac{\partial}{\partial r}\right) .
$$

In the POD technique, as in many other numerical methods employed for solving PDEs, the solution $T_{\text {prod }}$ is approximated by a $N$-terms truncated Fourier series of the form:

$$
T_{\text {prod }}(\xi, t) \approx \sum_{i=1}^{N} m_{i}(t) \phi_{i}(\xi),
$$

where $\xi$ represents the spatial coordinates $z$ and $r$. Basis functions in the set $\left\{\phi_{i}(\xi)\right\}_{i=1}^{N}$ are orthogonal and contain the spatial dependency of the solution, while the set $\left\{m_{i}(t)\right\}_{i=1}^{N}$ collects the time dependent coefficients. 
Each element $\phi_{i}(\xi)$ is computed off-line as the solution of the following integral eigenvalue problem (Sirovich, 1987; Ravindran, 2000):

$$
\int_{\mathbb{V}} R\left(\xi, \xi^{\prime}\right) \phi_{i}\left(\xi^{\prime}\right) \mathrm{d} \xi^{\prime}=\lambda_{i} \phi_{i}(\xi)
$$

where $\lambda_{i}$ corresponds with the eigenvalue associated with each global eigenfunction $\phi_{i}$. The kernel $R\left(\xi, \xi^{\prime}\right)$ in equation (B.3) corresponds with the two point spatial correlation function, defined as follows:

$$
R\left(\xi, \xi^{\prime}\right)=\frac{1}{\ell} \sum_{j=1}^{\ell} \hat{T}_{\text {prod }}\left(\xi, t_{j}\right) \hat{T}_{\text {prod }}\left(\xi^{\prime}, t_{j}\right),
$$

with $\hat{T}_{\text {prod }}\left(\xi, t_{j}\right)$ denoting the value of the field at each instant $t_{j}$. The values of $\hat{T}_{\text {prod }}\left(\xi, t_{j}\right)$ can be obtained experimentally or by direct numerical simulation of Eqn (B.1), and the summation extends over a sufficiently rich collection of uncorrelated snapshots at $j=1, \cdots, \ell$.

The number of elements $(N)$ in the approximation (B.2) is usually chosen using a criteria based on the so-called energy captured by the POD basis. Such term is related to the eigenspectrum $\left\{\lambda_{i}\right\}_{i=1}^{\ell}$ through the following expression:

$$
E(\%)=100 \times \frac{\sum_{i=1}^{N} \lambda_{i}}{\sum_{i=1}^{\ell} \lambda_{i}}
$$

5 The time dependent coefficients $\left\{m_{i}(t)\right\}_{i=1}^{N}$ in Eqn (B.2), are computed by projecting the original PDE system (B.1) into each element of the POD basis set. Formally this is done as follows:

$$
\int_{\mathbb{V}} \phi_{i} \frac{\partial T_{\text {prod }}}{\partial t} d \xi=\int_{\mathbb{V}} \phi_{i}\left(\alpha \mathcal{A} T_{\text {prod }}\right) d \xi ; \quad i=1, \ldots, N .
$$

758 Substituting the Fourier series approximation (B.2) into Eqn (B.6) leads to:

$$
\int_{\mathbb{V}} \phi_{i} \sum_{j=1}^{N} \phi_{j} \frac{d m_{j}}{d t} d \xi=\alpha \sum_{j=1}^{N} m_{j} \int_{\mathbb{V}} \phi_{i}(\mathcal{A}) \phi_{j} d \xi .
$$


The basis functions obtained from (B.3) are orthogonal and can be normalized so that:

$$
\int_{\mathbb{V}} \phi_{i} \phi_{j} d \xi=\left\{\begin{array}{lll}
1 & \text { if } i=j \\
0 & \text { if } i \neq j
\end{array} .\right.
$$

761 Eqn (B.7) is then rewritten as:

$$
\frac{\mathrm{d} \mathbf{m}}{\mathrm{d} t}=\alpha \mathbf{A m}
$$

${ }_{762}$ where $\mathbf{A}$ is the matrix resulting from the projection of the laplacian operator ${ }_{763} \mathbf{A}=\int_{\mathbb{V}} \Phi^{T} \mathcal{A} \Phi d \xi$ with $\Phi=\left[\phi_{1}, \phi_{2}, \ldots, \phi_{N}\right]^{T}$ and $\mathbf{m}$ corresponds with the ${ }_{764}$ following column vector $\mathbf{m}=\left[m_{1}, m_{2}, \cdots, m_{N}\right]^{T}$.

At this point both the basis functions and the time dependent coefficients are known and the field can be recovered by means of Eqn (B.2). Note that the accuracy of the approximation can be improved arbitrarily by increasing the number of elements $N$ in the basis set $\Phi$. 